Relations industrielles

Industrial Relations

\title{
Industrial Conflict and Dispute Settlement. Seventh Annual Conference (April 18-19, 1955). Montreal: Industrial Relations Centre, McGill University, 1955. 121 pp.
}

\section{Roger Chartier}

Volume 11, numéro 3, juin 1956

URI : https://id.erudit.org/iderudit/1022631ar

DOI : https://doi.org/10.7202/1022631ar

Aller au sommaire du numéro

Éditeur(s)

Département des relations industrielles de l’Université Laval

ISSN

0034-379X (imprimé)

1703-8138 (numérique)

Découvrir la revue

Citer ce compte rendu

Chartier, R. (1956). Compte rendu de [Industrial Conflict and Dispute Settlement. Seventh Annual Conference (April 18-19, 1955). Montreal: Industrial Relations Centre, McGill University, 1955. 121 pp.] Relations industrielles / Industrial Relations, 11(3), 220-221. https://doi.org/10.7202/1022631ar

Tous droits réservés (C Département des relations industrielles de l’Université Laval, 1956
Ce document est protégé par la loi sur le droit d'auteur. L'utilisation des services d'Érudit (y compris la reproduction) est assujettie à sa politique d'utilisation que vous pouvez consulter en ligne.

https://apropos.erudit.org/fr/usagers/politique-dutilisation/ 


\section{LIVRES ET REVUES}

Les recensions suivantes sont de Roger Chartier.

Disability Retirement in Industrial Pension Plans. By W. Michael Blumenthal. Princeton, N.J.: Industrial Relations Section, Princeton University, 1956, 62 pp.

Les publications de la Section de relations industrielles de Princeton sont rarement volumineuses, mais elles impressionnent par la netteté de leur présentation et nar le caractère scientifique de leur contenu. Celle-ci, de M. W. Machiel Blumenthal, s'intéresse au travailleur âgé et à l'âge de la retraite; plus précisément, elle étudie le problème des handicapés (disabled), qui, pour la plupart, ne sont pas - ou sont imparfaitement - protégés par les lois d'accidents de travail. M. Blumenthal, compilant les réponses faites à un questionnaire précis, donne le bilan de la protection accordée par 102 compagnies employant $3,000,000$ de travailleurs à ceux qui deviennent handicapés, temporairement ou non, totalement ou non, avant l'âge normal de la retraite. L'auteur en profite pour établir l'incidence de ces cas de «disability 》 avant l'âge de la retraite.

Formal Job Evaluation and Some of Its Economic Implications. By L. G. Nicolopoulos. Research Report No. 1. Montreal: The Industrial Relations Centre of MoGill University, 1954. 43 pp.

L'évaluation des tâches - ou qualification du travail - est à la morte dans l'industrie canadienne. Les plans se multiplient, imposants et souvent compliqués. La tentation de l'administrateur, en présence d'un tel appareil technique, est de s'enfermer dans la myopie et d'oublier de situer l'évaluation des tâches dans ses relations avec un monde plus large, celui des forces en interrelation sur le marché du travail.

Cette publication aux proportions modestes, qui s'appuie sur la pensée collective de techniciens du iob evaluation et d'économistes de McGill travaillant de concert, s'impose au lecteur par sa clarté et son sens critique. L'auteur y décrit l'évolution de la technique elle-même, les problèmes quotidiens que pose sa mise en application, l'attitude et la participation syndicales, les griefs que pose la. qualification du travail, ses avantages et inconvénients, et enfin, ses implications économiques.

Dans sa concision, ce petit livre repose d'énormes traités spécialisés d'où le sens critique est généralement absent.

\section{Industrial Conflict and Dispute Settle-} ment. Seventh Annual Conference (April 18-19, 1955). Montreal: Industrial Relations Centre, McGill University, $1955.121 \mathrm{pp}$.

Le Rapport du 7e Congrès des relations industrielles de McGill contient des conférences pour la plupart très intéressantes qui portent sur les conflits industriels et leur solution.

Celle du Dr H. D. Woods, directeur du Centre, contient une évaluation critique de l'arbitrage obligatoire au $\mathrm{Ca}$ nada. Ce texte stimulant a fait beaucoup de bruit, à une époque où, devant l'ampleur - bien souvent surfaite des malaises industriels, on disait à tort et à travers que la grève était une formule périmée, et qu'il convenait de laisser à un tribunal «impartial » le soin d'imposer aux parties la recette «la meilleure ». Le professeur Woods, après un relevé historique de la législation présente et de ses effets sur la négociation collective, suggérait au contraire qu'on laisse aux parties leur liberté tout au long du procédé; et que s'il devait y avoir changement, que celui-ci se fít dans le sens d'une plus grande latitude.

L'article du professeur Wilbert E. Moore, sociologue, sur la nature du conflit industriel, est bien ramassé, plein de concepts sociologiques, dans la veine de son Industrial Relations and the Social Order; Moore utilise également le nouveau volume édité par Kornhauser, Dubin et Ross sur I'Industrial Conflict, et celui de Gouldner sur le Wildcat Strike.

A souligner également larticle de David L. Cole sur l'art d'arriver à une entente dans la négociation collective; de l'hon. Milton Gregg sur les responsa- 
bilités sociales et le processus de conciliation; de J. C. Adams et de Eamon Park, sur l'attitude - de la direction et du syndicat, respectivement - à l'égard de la conciliation.

Municipal Labour Relations in Canada. By S. J. Frankel and R. C. Pratt. Montreal: The Industrial Relations Centre of McGill University and Canadian Federation of Mayors and Municipalities, 1954. 87 pp.fl $\$ 2.50$.

Ce petit texte, cartonné et très bien présenté, fait oeuvre de pionnier dans le domaine de la négociation collective sur le plan municipal. Il analyse la croissance du syndicalisme municipal, les problèmes de négociation collective des policiers et des pompiers, la législation provinciale qui régit les relations de travail au niveau municipal, la représentation municipale aux négociations; i! offre des critères logiques de niveaux de salaires; enfin, il aborde le problème de l'arbitrage obligatoire.

Près de 50,000 employés municipaux adhèrent à des syndicats qui négocient avec leur employeur. Ce chiffre, à lui seul, indique déjà l'importance de l'étude de Frankel et Pratt. Les auteurs ont résumé une matière difficile dans un texte clair, concis, critique. Leur étude de l'arbitrage, volontaire ou obligatoire, mérite particulièrement l'attention.

Sixth Annual Conference (April 21-22, 1954). Montreal: Industrial Relations Centre, McGill University, 1954, 125 pp., mimeo.

Pour qui cherche une source compacte et facile d'accès sur les problèmes des agents de maîtrise (contremaîtres, surintendants) dans l'industrie moderne, le. Rapport du 6e Congrès de McGill constitue une réponse partielle. Souligaons d'abord le texte serré, en deux sections, du professeur Kahn. de l'Université du Michigan, sur «Leadership Patterns and Organization Effectiveness». Puis, l'article très original et très stimulant du professeur Neil $W$. Chamberlain, quelque pøu dans la veine du précédent quant à l'obiet - le contremaître - sinon quant à l'« approche ». Se rattachant aux deux textes précités, la conférence du professeur Stagner, de l'Université de l'Illinois, souligne l'impontance des attitudes en relations indus- trielles; elle s'appuie sur les recherches très poussées que le professeur Stagner et son groupe ont effectuées à Illini City, et dont les résultats remplissent deux tomes imposants.

Deux autres conférences, qui font un peu figure de hors-d'oeuvre en dépit de leur valeur autonome, viennent compléter le volume. La première, du professeur Kierans, décrit les \& Full Employment Stresses 》; la seconde, de M. George W. Brooks, un chef syndical, traite des avantages et limitations de la convention collective.

\section{Effective Personnel Selection Procedures. \\ By C. Harold STONE and William E.}

KENDALL. Englewood Cliffs, N.J.:

Prentice-Hall, Inc., 1956. 433 pp.

Both authors are psychologists actively working in the Personnel field, and this shows in the down-to-earthness of their approach and in the step-by-step techniques which are described alongside more lofgty statements of principles and policies. Emphasis, it seems, has been placed on research and evaluation of programs, practices and procedures used in companies.

The book deals with the ways and means of finding people and putting them to work. Over this extravagantly vast array of textbooks discussing with considerable superficialness, doubtless - all the many facets of Personnel Administration, Stone and Kendall's book has the definite advantage of bringing together into one volume all major topics of recruitment, selection and induction.

Part III, on Testing, is especially recommended.

The book, although competent, makes no attempt at sophistication. It is a well-written, well-subdivided textbook of an introductory nature covering quite thoroughly the selection process in Personnel work. Students are likely to find it most useful; practicioners will read it as a good refresher.

\section{Handbook of Industrial Engineering and} Management. Edited by W. Grant Ireson and Eugene L. Grant. Englewood Cliffs, N.J.: Prentice-Hall, Inc., $1955, \quad 1203$ pp. $\$ 12.00$.

Inutile, en quelques lignes, de tenter de rendre justice à ce mammouth aux 